\title{
RELACIÓN ENTRE LA PERCEPCIÓN DEL RIESGO BIOLÓGICO Y LA ACCIDENTALIDAD LABORAL EN UN HOSPITAL COLOMBIANO, 2019
}

\begin{abstract}
Johny Andrés Uribe Salazar ${ }^{1}$, Oscar Augusto Bedoya Carvajal², Diego Enrique Vélez Gómez³
${ }^{1}$ Bacteriólogo y Laboratorista Clínico. Universidad Colegio Mayor de Antioquia. Medellín, Colombia. Correo electrónico: jandresuribes22@gmail.com

2MSc Salud Pública. Docente e investigador Universidad Remington. Medellín, Colombia.

${ }^{3}$ Administrador en Salud, Gestión Sanitaria y Ambiental. Facultad Nacional de Salud Pública, Universidad de Antioquia, Medellín, Colombia.

\section{RESUMEN}

La percepción del riesgo está asociada a la ocurrencia de accidentes laborales. Para las empresas de salud es fundamental conocer este fenómeno, favoreciendo así la implementación de estrategias de afrontamiento y prevención, que contribuyen en la disminución de la accidentalidad laboral. Objetivo: Analizar la percepción del riesgo biológico y los factores asociados a la accidentalidad laboral a través del método EPRO (Evaluación de Percepción de Riesgo Ocupacional) y el software RISKPERCEP de los empleados de un hospital de Antioquia, Colombia, en el año 2019. Metodología: Estudio explicativo transversal que empleó la metodología EPRO y el software RISKPERCEP a través de una encuesta estructurada a todos los trabajadores $(\mathrm{N}=50)$ de la institución. La información estadística se analizó con el software SPSSv24. Resultados: Se encuentra un perfil de subestimación del riesgo biológico laboral en las diferentes áreas de la institución. A la accidentalidad laboral se asocian factores como el tiempo desarrollando la misma actividad y la percepción del estado de salud. Conclusión: Los trabajadores con mayor experiencia y exposición al riesgo biológico laboral subestiman el riesgo.
\end{abstract}

Palabras clave: Riesgo; Percepción del riesgo; Riesgo biológico; Evaluación de percepción de riesgo; Accidente laboral; RISKPERCEP.

Recibido: 27 de Mayo de 2020. Aceptado: 14 de Diciembre de 2020

Received: May 27, $2020 . \quad$ Accepted: December 14, 2020

\section{RELATIONSHIP BETWEEN THE PERCEPTION OF BIOLOGICAL RISK AND WORK ACCIDENTALITY IN A COLOMBIAN HOSPITAL, 2019}

\begin{abstract}
The perception of risk is associated with the occurrence of occupational accidents. For health companies it is fundamental to know this phenomenon, thus favoring the implementation of coping and prevention strategies, which contribute to the reduction of work accidentality. Objective: To analyze the perception of biological risk and the associated factors with occupational accidents through the ORPE (Occupational Risk Perception Evaluation) method and the RISKPERCEP software of the employees of a hospital in Antioquia, Colombia, in 2019. Methodology: Cross-sectional explanatory study that used the ORPE methodology and the RISKPERCEP software through a structured survey of all the workers $(\mathrm{N}=50)$ of the institution. Statistical information was analyzed with SPSSv24 software. Results: An underestimation profile of occupational biological risk is found in the different areas of the institution. Factors such as time developing the same activity and perception of the state of health are associated with work accidentality. Conclusion: Workers with more experience and exposure to occupational biological risk underestimate the risk.
\end{abstract}

Keywords: Risk; Risk perception; Biological risk; Risk perception evaluation; Work accident; RISKPERCEP.

Cómo citar este artículo: J. Uribe, O. Bedoya, D. Vélez . "Relación entre la percepción del riesgo biológico y la accidentalidad laboral en un hospital Colombiano, 2019”, Revista Politécnica, vol.16, no.32 pp.56-67, 2020. DOI:10.33571/rpolitec.v16n32a5 


\section{INTRODUCCIÓN}

El riesgo biológico laboral es definido como la probabilidad de adquirir cualquier infección, alergia o toxicidad que pueda ser causada por microorganismos en el lugar de trabajo. [1] [2]

Los profesionales de la salud que más se exponen a dicho riesgo son los que están en áreas asistenciales y de laboratorio. La inadecuada manipulación de elementos cortopunzantes es la principal causa de accidentalidad laboral por riesgo biológico [3] [4], el subregistro de este evento es de aproximadamente el $41 \%,[5]$ [6] siendo uno de los principales factores de riesgo pese a la existencia de guías y protocolos nacionales e internacionales que pretenden ayudar a controlar este peligro biológico. [2]

Cada año en el mundo se estiman unas 320.000 muertes como consecuencia de la transmisión de enfermedades infecciosas por accidentes laborales y de 5.000 muertes/año en la Unión Europea. [7]

En España se reporta que cerca de la mitad de los accidentes laborales ocurrieron a causa de un exceso de confianza o tolerancia al riesgo, [8] otros estudios reportan que el $85 \%$ de los casos estuvieron relacionados con actos inseguros y no fueron generados por condiciones de inseguridad del medio. [9]

En Colombia para el año 2018, el 7\% ( $n=31.631)$, de los afiliados a las ARL en el sector "servicios sociales y de salud" sufrieron de un accidente laboral calificado, correspondiendo al $16,9 \%$ de la totalidad de accidentes laborales registrados. Así mismo, entre las 3.686 enfermedades laborales calificadas a nivel nacional, el $11,6 \%$, correspondieron al sector salud. [10]

Los accidentes laborales con exposición a material biológico suponen un riesgo de infección, el cual puede acarrear consecuencias graves e irreversibles para la salud del trabajador, como el VIH (Virus de Inmunodeficiencia Humana), Síndrome de Inmunodeficiencia Adquirida (SIDA), VHB (Virus de la Hepatitis B), VHC (Virus de la Hepatitis C), VHD (Virus de la Hepatitis Delta) u otros microorganismos como bacterias, parásitos, hongos patógenos y cultivos celulares. [11]

Es fundamental liderar las acciones de seguridad y salud en el trabajo (SST) y evaluar la percepción del riesgo biológico desde la alta gerencia, teniendo un enfoque de la cultura y valores organizacionales [12] con fin de favorecer la implementación de estrategias de afrontamiento y prevención, mejorar el Sistema de Gestión de Seguridad y Salud en el Trabajo (SGSST), fortalecer las normas de bioseguridad, [13] estructurar y generar estrategias de promoción de la salud y prevención de la enfermedad ( $\mathrm{P}$ y $\mathrm{P}$ ), fortalecer las campañas de autocuidado y mejorar los sistemas de vigilancia epidemiológicos en las instituciones.

Esto contribuye al desarrollo de entornos laborales saludables [14], generando un impacto positivo en la salud y calidad de vida de los trabajadores al disminuir las incapacidades por accidentes laborales, lo que a su vez se ve reflejado en la productividad de las instituciones y la reducción de los costos directos e indirectos de la accidentalidad laboral por este tipo [15] [16] beneficiando así al sistema de salud y permitiendo contar con protocolos y guías más efectivas en el tema. [17] [18]

\section{Percepción del riesgo laboral}

La percepción del riesgo es definida como el reconocimiento de la capacidad del peligro para hacer daño y como estimación de la probabilidad de que ocurra ese evento y las posibles consecuencias que conllevaría. [19] [8] Esta se da a partir de los estímulos que son captados por los sentidos, los cuales son analizados e interpretados, teniendo como referente los valores y aspectos culturales que dan significado al sujeto [20] y puede ser abordada con técnicas psicométricas que emplean análisis multivariantes y escalonados, dándose una evaluación dimensional. [21] [22]

La base para comprender la percepción del riesgo desde una mirada integral comprende la influencia de tres factores (individuales, sociales y organizacionales) en las decisiones que toman las personas frente a situaciones de peligro en su área de trabajo, permitiendo así, contar con una valoración del riesgo más objetiva y acercada a la realidad. [23] 
Los factores individuales hacen referencia a las actitudes, prácticas, conductas, aspectos cognitivos, conocimientos adquiridos y la experiencia laboral, [24] los organizacionales a la cultura organizacional de las instituciones y los factores sociales a patrones culturales, como costumbres, ideas y creencias, [21] así como diferentes factores del medio en el que interactúa el ser humano. [25] [26] La percepción del riesgo es un componente importante asociado a los errores humanos [27] e involucrado en la ocurrencia de accidentes laborales. [28]

Dada las situaciones expuestas anteriormente, se evidencia una problemática que enmarca a los trabajadores del sector salud, donde es necesario conocer relación que existe entre la percepción del riesgo biológico y la accidentalidad laboral, por lo que el presente estudio evaluó la percepción del riesgo biológico laboral en un hospital de primer nivel de Antioquia, Colombia.

Los resultados brindan evidencia para que las instituciones de salud estructuren y generen estrategias de promoción de la salud y prevención de la enfermedad ( $P$ y P), fortalezcan las campañas de autocuidado desde la salud y seguridad en el trabajo, que permitan apalancar y mejorar los sistemas de vigilancia epidemiológicos en las instituciones.

\section{MATERIALES Y METODO}

Estudio explicativo transversal que utilizó como fuente de información primaria los datos recolectados con el instrumento RISKPERCEP de los empleados de un hospital del departamento de Antioquia, Colombia. La población objeto fue la totalidad (censo) de los empleados de dicha institución $(\mathrm{N}=50)$. El estudio contó con la aprobación del comité de ética de dicha institución y cada participante firmó un consentimiento informado.

Se partió de un enfoque cuantitativo, que se basó en el método EPRO a través del software RISKPERCEP cuya herramienta es psicométrica y específica para este tema, la cual se emplea para medir la percepción del riesgo con la aplicación de encuestas, utilizando instrumento de recolección de información estructurado y específico para la medición de la percepción del riesgo biológico [29]

Utiliza variables politómicas ordinales, las cuales tienen como objeto "fragmentar" el pensamiento, para analizarlo en un contexto de factores independientes [30] [31]. Las variables empleadas son de tres tipos, de carácter individual, naturaleza del riesgo y gestión del riesgo, que indagan por aspectos como la familiaridad del sujeto con la situación de riesgo, comprensión del riesgo, capacidad de control, edad, ingresos, vinculación laboral y clima organizacional. [32]

\section{Técnicas de Recolección de Información}

Se aplicó el instrumento de recolección de información (encuesta) en formato físico a los trabajadores de la institución, previo diligenciamiento del consentimiento informado.

Una vez se contó con la totalidad de las encuestas diligenciadas, se digitó la información en el software Microsoft Excel, en el cual se construyó una base de datos, esta se exportó al software RISCKPERCEPC versión industrial (licencia adquirida por el investigador) y al software estadístico SPSS versión 24 (licenciado por el Politécnico Jaime Isaza Cadavid).

Al exportar la base de datos al software RISCKPERCEP se corrieron los análisis de percepción del riesgo biológico de manera grupal, individual y por dimensiones (factores individuales, de naturaleza y gestión del riesgo). Se generó un estimador "Score ponderado de percepción", dando una cifra exacta del riesgo percibido para cada trabajador entre 0 y 5. Los valores menores a 2,0 denotan una subestimación del riesgo, entre 2,1 y 3,0 una adecuada estimación y los valores mayores a 3,0 una sobreestimación del riesgo. [32] [29]

Al exportar la base de datos al software estadístico SPSS se generaron los análisis univariados (estadística descriptiva), bivariados (cruce de variables y asociaciones entre estas) y análisis multivariado (modelo explicativo de regresión logística).

Para establecer la asociación entre dos variables cualitativas se aplicó la prueba de Chi-cuadrado $\left(\mathrm{X}^{2}\right)$, se consideró un valor $\mathrm{p} \leq 0,05$ como estadísticamente significativo, se estimó el riesgo para la accidentalidad laboral mediante la razón de oportunidades (OR) con intervalos de confianza del 95\%. 
Para el análisis multivariado se realizaron regresiones logísticas con aquellas variables que en los diferentes análisis bivariados tuvieron una asociación con un valor de $p \leq 0,25$, logrando así llegar a un modelo explicativo entre la percepción del riesgo biológico y la accidentalidad laboral.

\section{RESULTADOS}

La población del hospital presentó una distribución por sexo, en la que aproximadamente tres cuartas partes de esta son mujeres, con un $76 \%(\mathrm{~N}=38)$ y un $24 \%$ de hombres $(\mathrm{N}=12)$, para un total de 50 participantes.

El $64 \%$ de los trabajadores pertenecía al área asistencial y el $36 \%$ al área administrativa. El $46 \%$ tenían una formación técnica, seguido por un 34\% universitario y un 8\% educación básica (primaria y bachiller).

El $80 \%$ de los trabajadores tenía menos de 47 años de edad, el $50 \%$ de los hombres y un $38,9 \%$ de las mujeres tenía entre 1 y 5 años de experiencia. El 10,4\% de la población estudiada contaba con 1 año o menos de tiempo de experiencia laboral, el $41,7 \%$ entre 1 a 5 años y el $47,9 \%$ más de 5 años. Al hacer el análisis por áreas, el 5,9\% de la población del área administrativa tenía menos de un año de experiencia, mientras que en el área asistencial fue del 12,9\%.

La utilización de elementos de protección personal marcó una diferencia según el tiempo de experiencia laboral de cada trabajador, donde quienes tenían 5 años o menos de experiencia laboral, utilizaban en un $88,2 \%$ guantes y gafas, y en un $82,4 \%$ dental; proporciones superiores al grupo de mayor experiencia.

Es relevante contrastar que el $72 \%$ considera que tenía capacitación suficiente en el tema de riesgo biológico, pero al indagar por el conocimiento de las medidas para afrontar este riesgo, menos de la mitad de los empleados $(34,7 \%)$ conocía sobre estas, en el área administrativa la proporción fue del $22,2 \%$ y en el área asistencial del $41,9 \%$, reflejando así debilidad en las capacitaciones que el personal ha recibido y encontrando una oportunidad de mejora para la institución en cuanto a las capacitaciones que ofrece sobre el tema.

El 34\% de la población, afirmó tener poco o ningún conocimiento relacionado con el concepto de riesgo biológico, se encontró que el en área asistencial fue de un $25 \%$ y en el área administrativa esta proporción aumentó al 50\%.

Si los empleados tuvieran un accidente de trabajo de tipo biológico, el $28 \%$ no conocerían las consecuencias post exposición a este riesgo y el $24 \%$ tendrían poco conocimiento.

Cerca de la mitad de la población (44,9\%) consideró que puede mantener todos los riesgos del puesto de trabajo bajo control, los hombres nunca consideraron tener control de la totalidad de los riesgos en ambas áreas, en cambio, el 66,7\% de las mujeres del área administrativa y el 54,5\% del área asistencial consideraron que mantenían control en todos los riesgos.

El $74 \%$ de todos los empleados del hospital consideraron que las consecuencias post exposición al riesgo biológico laboral pueden afectar la salud de su grupo familiar, el $94,4 \%$ de los trabajadores del área administrativa y el $65,2 \%$ de los trabajadores del área asistencial consideraron esto y un $21,7 \%$ afirmaron no tener conocimiento sobre el tema en mención.

El 45,8\% de la población manifestó que aceptaría realizar alguna actividad de sobreexposición a los riesgos laborales si recibiera algún tipo de remuneración y/o bonificación por realizar dicha actividad, se encontró un comportamiento similar entre las dos áreas (administrativa y asistencial).

Hubo una mayor proporción de trabajadores que aceptaría algún tipo de bonificación o remuneración para sobrexponerse a los riesgos en el grupo que tenía 5 años o menos de experiencia. Se evidencia así que entre más años de experiencia menor es la utilización de elementos de protección de personal, considerando un grado de confianza alto con el entorno de trabajo.

\section{Accidentalidad laboral}

La institución reportó 11 accidentes laborales, lo cual corresponde al 22\% de sus trabajadores. Esto durante 18 meses, entre enero de 2018 y julio de 2019. Es importante mencionar que la totalidad de accidentes laborales reportados correspondieron al área asistencial. El área administrativa no presentó accidentes 
laborales en los últimos dos años evaluados, observándose una asociación estadísticamente significativa entre la accidentalidad y el área. $(p=0,003)$. En el área asistencial hubo una accidentalidad del $44,4 \%$ de los hombres y del $30,4 \%$ de las mujeres.

La accidentalidad en los dos años evaluados se concentró en aquellos trabajadores que contaban con cinco años o menos de experiencia laboral, teniendo una proporción del $36 \%$ frente al $8,7 \%$ en el grupo de trabajadores con mayor tiempo de experiencia laboral (>6 años), presentando una asociación estadísticamente significativa con la accidentalidad, $(p=0,025)$, dado que el $81,8 \%$ de los accidentes que se presentaron fueron en los trabajadores que tenían 5 años o menos de experiencia. Este aspecto guarda relación con la variable que indaga por el tiempo que la persona lleva realizando la misma actividad laboral, donde se observó que el $90 \%$ de accidentes ocurridos pertenecieron a personas que llevaban 5 años o menos realizando la misma actividad, esta relación fue estadísticamente significativa $(p=0,019)$.

También se encontró que un $37 \%$ de los trabajadores entre 18 y 30 años de edad sufrieron un accidente laboral en los dos últimos años, mientras que en el grupo de trabajadores entre 31 y 59 años de edad la proporción de accidentalidad fue menor, con un $4,3 \%$.

Es llamativo encontrar que, del total de trabajadores que presentaron un accidente, ninguno tuviera hijos, mientras que en el grupo que no tenía hijos, hubo una accidentalidad laboral del 32,4\%. (Asociación estadísticamente significativa con un valor $\mathrm{p}=0,008$ ).

Al indagar por los riesgos laborales que los trabajadores consideraron tener bajo control en su puesto de trabajo, se encontró que quienes consideraban tener algunos de estos bajo control presentaron una accidentalidad del $30,4 \%$, mientras que los trabajadores que afirmaron que no podían tener bajo control ningún riesgo, es decir quienes presentaron menos tolerancia al riesgo, no se accidentaron.

A menor tiempo de experiencia laboral se presentó mayor accidentalidad, dado que más de la mitad (60\%) de personas en el grupo de quienes contaban con un año o menos de experiencia presentó accidentes, seguido del grupo con experiencia entre 1 a 5 años (30\%) y por último el grupo de con 5 años o más de experiencia, con un $8,7 \%$ de accidentalidad.

Aquellos trabajadores que siempre estarían dispuestos a tener una sobreexposición a cualquier tipo de riesgo laboral presentaron mayor proporción de accidentalidad laboral $(33,3 \%)$ respecto a las personas que nunca estarían dispuestas a tener dicha exposición (23,1\%) o los que aceptarían esto algunas veces (19\%).

\section{Percepción de riesgo}

El 58\% de la población presentó una subestimación del riesgo y el 42\% una adecuada estimación a este. Ninguna persona mostró una sobreestimación del riesgo. En la figura 1 se puede observar el comportamiento de la percepción de riesgo entre el personal asistencial y administrativo entre los diferentes grupos de variables analizados.

La media general para la percepción del riesgo fue de 1,952 (Subestimación del riesgo), este valor fue más bajo en el área asistencial $(1,927)$. Hubo una diferencia de 0,025 puntos entre las áreas, sin modificar su calificación, ubicándolas en subestimación del riesgo. (la subestimación del riesgo se da en el rango entre 0 y 2).

La desviación estándar global es de 0,179 , esto indica que la mayor parte de los datos tienden a estar agrupados cerca de la media, entre valores que oscilan entre 2,132 y 1,773, es decir en el límite entre subestimación del riesgo y adecuada estimación, teniendo en cuenta que el rango para la calificación de estimación adecuada es entre 2,1 y 3,0 .

En la figura 1 se puede apreciar que fueron más notables las subestimaciones por las variables de "familiaridad" [FAMI] las cuales denotan el grado de experiencia del sujeto con la situación, las variables de "mayor sentimiento de control" [CONT], referentes al grado en que el sujeto puede ejecutar una conducta efectiva para modificar la situación de riesgo y las variables de "alta demanda asistencial" [DEMA] referentes a la influencia del ritmo de trabajo, horario y condiciones laborales sobre la atención, siendo el grupo más expuesto directamente a los riesgos. 


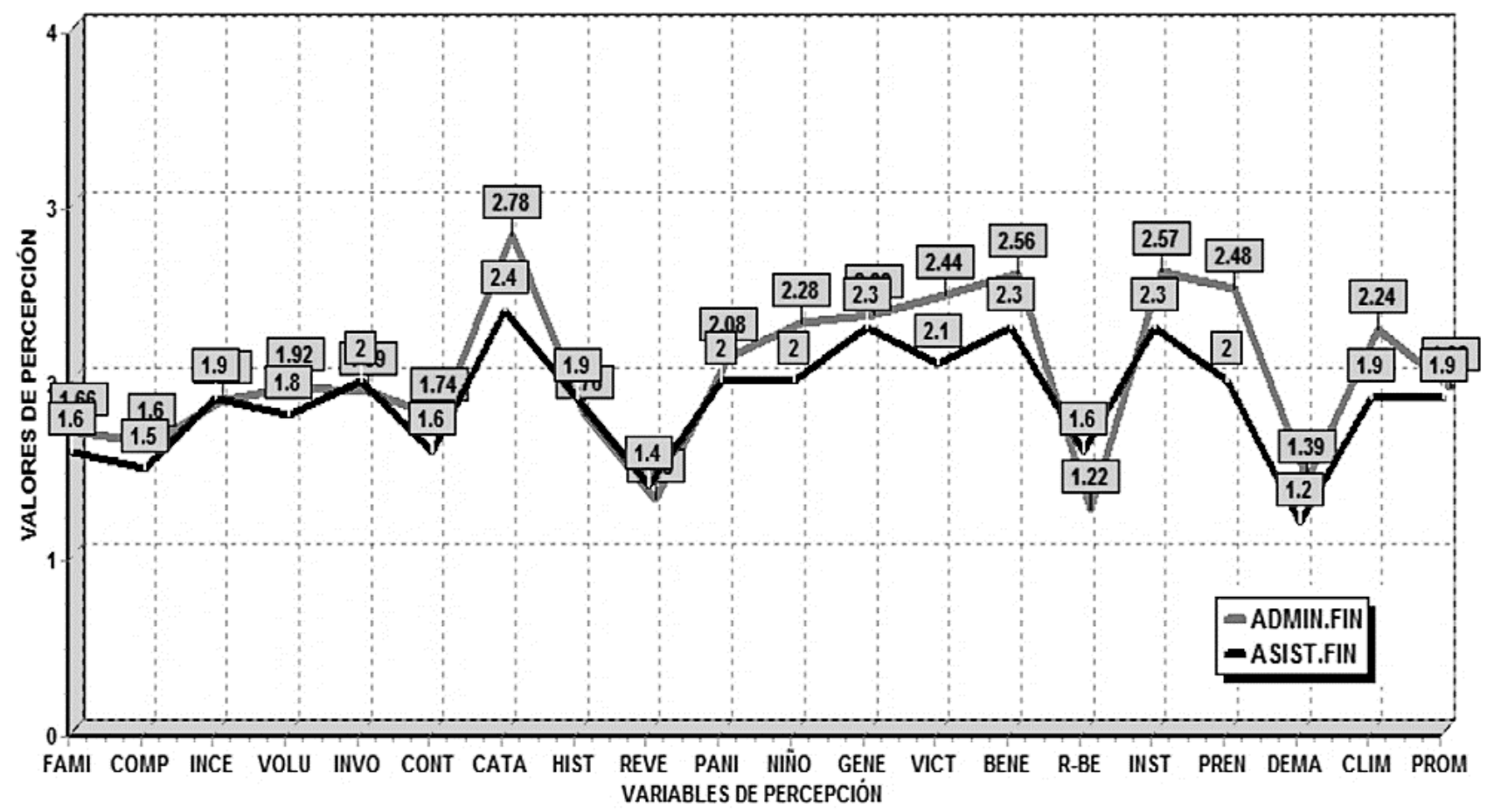

Figura 1. Comparativo entre áreas administrativo (línea gris) y asistencial (línea negra) por RISKPERCEP

Esto muestra que existe una tolerancia a los riesgos y se está generando una subestimación de estos. Otro aspecto en donde se encontró diferencia fue entre los grupos de edad, existiendo una mayor subestimación del riesgo en aquellas personas que tienen entre 18 y 30 años de edad, con un $63 \%$, mientras que en el grupo de personas entre los 31 y 59 años de edad la subestimación disminuye al $52,2 \%$. Si bien, más de la mitad de la población en los dos grupos presentó una subestimación, es recurrente observar menor "adecuada estimación" del riesgo en las personas más jóvenes.

En aquellas personas que tenían 5 años o menos de experiencia, se encontró una subestimación del riesgo en un $60 \%$, y en las que contaban con mayor experiencia (6 años o más) una subestimación del $56,5 \%$.

El 55,6\% de trabajadores en el área administrativa tuvo una adecuada estimación del riesgo, y en una menor proporción los trabajadores del área asistencial $(34,4 \%)$.

Al calcular la asociación entre las variables de percepción y la accidentalidad laboral con la prueba de chi cuadrado y la estimación de la razón de oportunidades Odds ratio (OR), se encontró asociación entre la edad y la accidentalidad laboral $(p=0,006)$, donde los trabajadores con edades entre 18 y 30 años presentaron 12,941 veces más probabilidades de tener un accidente laboral que los que tenían entre 31 y 59 años de edad. (Ver tabla 1).

También aquellos trabajadores que manifestaron llevar menos de 5 años desarrollando la misma actividad tuvieron 9,474 veces más probabilidad de accidentarse frente a los que llevaban más de 5 años desarrollándola $(\mathrm{p}=0,019)$.

Se observó que existe asociación estadísticamente significativa entre el tiempo de experiencia laboral y la accidentalidad $(p=0,026)$, donde aquellos trabajadores con menos de 5 años de experiencia presentaron 5,906 veces más probabilidad de sufrir un accidente que aquellos que contaban con más de 6 años de experiencia.

Tabla 1. Factores asociados a la accidentalidad laboral (OR crudo) 


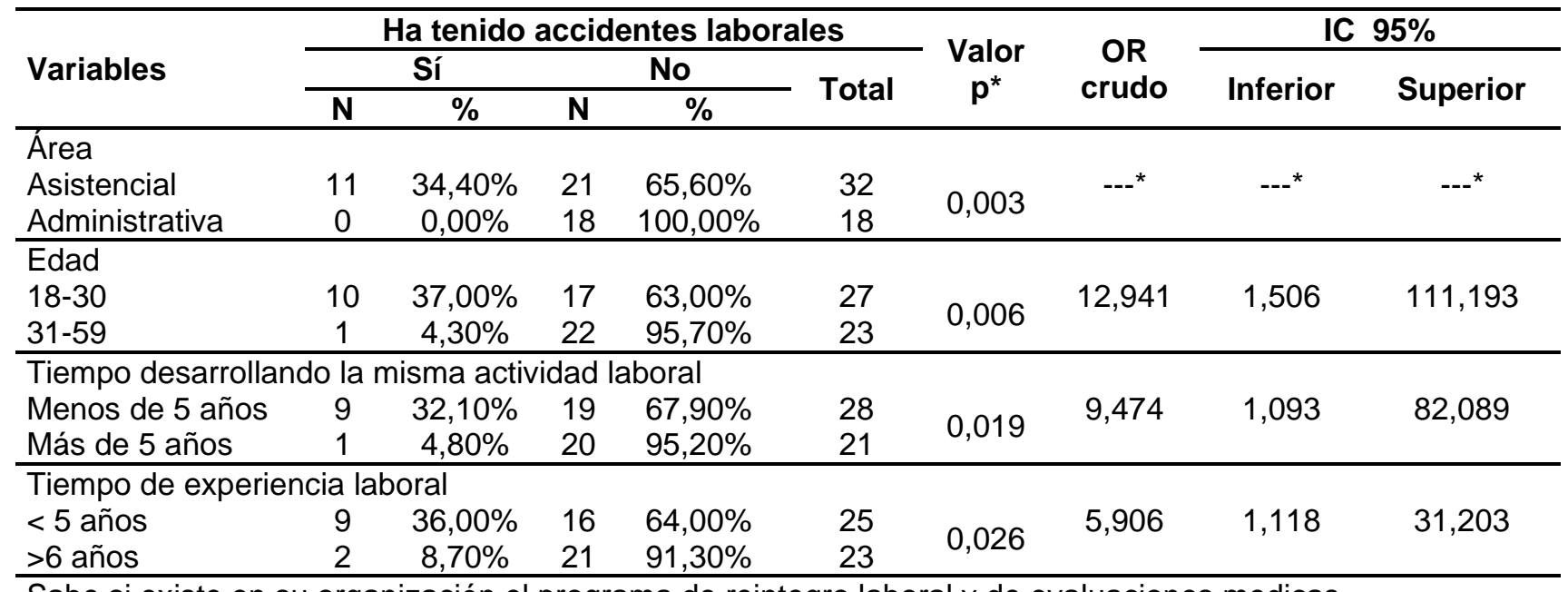

Sabe si existe en su organización el programa de reintegro laboral y de evaluaciones medicas ocupacionales

\begin{tabular}{|c|c|c|c|c|c|c|c|c|c|}
\hline $\begin{array}{l}\text { No Conoce } \\
\text { Si Conoce }\end{array}$ & $\begin{array}{l}6 \\
5 \\
\end{array}$ & $\begin{array}{l}42,90 \% \\
13,90 \% \\
\end{array}$ & $\begin{array}{c}8 \\
31 \\
\end{array}$ & $\begin{array}{l}57,10 \% \\
86,10 \% \\
\end{array}$ & $\begin{array}{l}14 \\
36 \\
\end{array}$ & 0,026 & 4,650 & 1,125 & 19,212 \\
\hline \multicolumn{10}{|c|}{ Años tiene de experiencia en la profesión } \\
\hline Menos de 5 años & 9 & $\begin{array}{c}33,30 \% \\
870 \%\end{array}$ & $\begin{array}{l}18 \\
21\end{array}$ & $66,70 \%$ & 27 & 0,037 & 5,250 & 1,002 & 27,514 \\
\hline \multicolumn{10}{|c|}{ Considera que las medidas de seguridad en su trabajo cubren todos los peligros } \\
\hline Si considera & 10 & $28,60 \%$ & 25 & $71,40 \%$ & 35 & \multirow[t]{2}{*}{0,085} & 5,600 & \multirow[t]{2}{*}{0,648} & \multirow[t]{2}{*}{48,424} \\
\hline No considera & 1 & $6,70 \%$ & 14 & $93,30 \%$ & 15 & & & & \\
\hline
\end{tabular}

Considera que en algunos casos le consume tiempo o le resulta incómodo el cumplimiento de normas de seguridad

$\begin{array}{llllllllll}\text { No considera } & 2 & 10,00 \% & 18 & 90,00 \% & 20 & 0,090 & 3,860 & 0,740 & 20,210 \\ \text { Si considera } & 9 & 30,00 \% & 21 & 70,00 \% & 30 & 0,090\end{array}$

Recibe alguna remuneración adicional por las funciones que realiza en la organización

$\begin{array}{llllllllll}\text { No recibe } & 7 & 17,50 \% & 33 & 82,50 & 40 & 0,135 & 0,320 & 0,070 & 1,430\end{array}$

$\begin{array}{lllllll}\text { Sí recibe } & 4 & 40,00 & 6 & 60,00 & 10 & 0,135\end{array}$

Percepción del estado de salud

$\begin{array}{llllllllll}\text { Muy buena } & 10 & 30,30 \% & 23 & 69,70 \% & 33 & 0,105 & 5,220 & 0,600 & 45,740\end{array}$

$\begin{array}{lllllll}\text { Regular } & 1 & 7,70 \% & 12 & 92,30 \% & 13 & 0,105\end{array}$

5 se analizó el valor de Pearson.

---* Sin determinar por tener valores en cero.

Finalmente, para determinar los factores que mejor explicaban la accidentalidad, se construyó un modelo de regresión logística binaria con todas aquellas variables que mostraron una asociación con un valor $p<0,25$ (siguiendo el criterio de Hosmer Lemeshow). [33]

Se encontró que las personas con 5 años o menos de experiencia laboral tuvieron 11,95 veces más probabilidad (OR ajustado) (IC 95\% 1,079-132,379) de presentar un accidente laboral de tipo biológico, que aquellos con más de 5 años de experiencia. $p=(0,043)$. (Ver tabla 2$)$.

Con una menor significancia estadística $(>0,05)$, se encontró que quienes consideraban que el cumplimiento de normas de seguridad les consumía tiempo o les resultaba incómodo $(p=0,067)$ tuvieron 11,081 veces más probabilidad de presentar un accidente laboral (IC 95\% 0,849-144,709), además quienes tenían una percepción del estado de salud muy buena presentaron un OR de 5,79 (IC 95\% 0,408-82,079) ( $p=0,194)$, como puede ser observado en la tabla 2.

Tabla 2. Factores asociados a la accidentalidad laboral (OR ajustado) 


\begin{tabular}{lccc}
\hline \multicolumn{1}{c}{ Factor asociado } & Valor $\mathbf{p}^{*}$ & $\begin{array}{c}\text { OR } \\
\text { ajustado }\end{array}$ & IC 95\% \\
\hline Menos de 5 años desarrollando la misma actividad laboral & 0,043 & 11,95 & $1,08-132,38$ \\
$\begin{array}{l}\text { Considera que en algunos casos le consume tiempo o le } \\
\text { resulta incómodo el cumplimiento de normas de seguridad }\end{array}$ & 0,067 & 11,081 & $0,85-144,71$ \\
$\begin{array}{l}\text { Percepción del estado de salud muy buena } \\
\begin{array}{l}\text { No recibe alguna remuneración adicional por las funciones } \\
\text { que realiza en la organización }\end{array}\end{array}$ & 0,194 & 5,797 & $0,41-82,08$ \\
\hline
\end{tabular}

*Prueba Wald

\section{DISCUSIÓN}

Ana teresa Carbonell y colaboradores, [23] al realizar un estudio de evaluación de percepción del riesgo en diversos escenarios con peligro biológico asociado, obtuvo un perfil de subestimación de los peligros entre el personal expuesto, guardando similitud en lo encontrado en el presente estudio, donde se encontró una subestimación del riesgo en ambas áreas (administrativa y asistencial), en un 58\% de la población.

En el estudio de Carbonell, [23] entre los técnicos de nivel medio y universitarios se encontró mayor percepción del riesgo que entre los obreros, los autores afirman que esto se debe a su mayor preparación y participación en las decisiones de la entidad, caso contrario a lo encontrado en el hospital, donde la población que goza de un nivel educativo alto presentó una mayor subestimación del riesgo, quizá debido a la tolerancia al riesgo adquirida por la experiencia y el tiempo desarrollando la misma actividad.

Entre las variables de percepción, la confianza en la institución estuvo dentro de las que tuvieron mayor índice de sobrestimación del riesgo, al igual que en el hospital, donde esta tuvo un valor de 2,30 (en la escala RISKPERCEP).

En el trabajo realizado por soler y colaboradores, [27] sobre la evaluación de percepción de riesgo, se emplea el mismo software (RISKPERCEP), esta vez en trabajadores de radioterapia, la investigación permite apreciar una subestimación del riesgo con un valor global promedio de 1.97, similar a lo encontrado en el hospital colombiano $(1,952)$ siendo inferior a 2 en ambos casos, mostrando una subestimación del riesgo, motivada esencialmente por el bajo conocimiento o comprensión de los riesgos. En esta investigación, los trabajadores presentaron un buen conocimiento de las acciones de divulgación dentro de la empresa, aspecto diferente al hospital colombiano. [27]

De manera general, los investigadores concluyen en que los errores humanos son los principales causantes de accidentes laborales, dado a que estas personas tienen una mayor subestimación del riesgo, que origina apatía y optimismo irracional, como a la sobreestimación del riesgo, que provoca alta tensión, estrés y pesimismo. [23] [27]

En otro estudio, González [24] utiliza un método diferente para evaluar la percepción del riesgo en el sector de la construcción, el cual maneja dos instrumentos de medición (paradigma psicométrico) diferente al RISKPERCEP, arrojando resultados en una escala de 0 a 5 para cada actividad desarrollada, mientras que RISKPERCEP tiene una metodología validada, con una única encuesta y software de apoyo para su análisis, lo que puede mejorar los tiempos de medición y generación de resultados. Además, ofrece una calificación global y por dimensiones a diferencia del método que utilizo González, donde se da una calificación para cada una de las actividades que realiza el trabajador.

En el estado de Baja California - México, se realizó un estudio de percepción del riesgo en salud por exposición a mezclas de contaminantes en personas que viven cerca de corrientes de agua. [34] A pesar de que las personas trabajan en contacto con sustancias tóxicas y peligrosas, la percepción del riesgo sobre la salud por exposición ocupacional y ambiental fue baja, donde la población no percibió que está expuesta permanentemente a sustancias peligrosas, lo cual disminuyó su percepción, aspecto similar a la situación encontrada en el hospital colombiano, en donde los empleados del área asistencial mostraron mayor confianza 
al riesgo y son quienes estaban expuestos permanentemente a riesgo biológico presentaron mayor accidentalidad laboral.

Aunque la población participante en los dos estudios es diferente en cuestión de riesgos laborales y entorno en el que se encuentran, se observa semejanza en cuanto a la confianza frente al riesgo, jugando esto un papel importante en la percepción del riesgo laboral en diferentes áreas.

Otros autores afirman que dada la presión por un mejor desempeño, los trabajadores tienden a adoptar prácticas de trabajo inadecuadas, [35] esto evidencia una problemática que enmarca a los trabajadores del sector salud, presentando tasas de accidentalidad representativas. [10]

Se encuentra así, una relación entre el estudio realizado en el hospital colombiano y los estudios desarrollados en otros países sobre la percepción del riesgo biológico laboral y otros riesgos empleando el RISKPERCEP y otros métodos.

El presente estudio bases para ayudar a intervenir el fenómeno desde la creación de estrategias seguras de prevención y control, enfocar acciones educativas y de mitigación para afinar los planes de promoción de la salud y prevención de la enfermedad y disminuir así las enfermedades profesionales asociadas al riesgo biológico laboral, las cuales pueden traer consecuencias para la salud de las personas. Dentro de los beneficios que puede traer para las instituciones está el disminuir las incapacidades por accidentes laborales de riesgo biológico, aumentando así el bienestar de sus trabajadores y disminuyendo costos al sistema.

Las acciones de fortalecimiento a los diferentes sistemas podrían ser aplicados en diferentes instituciones prestadoras de salud (IPS), lo que hace del estudio un insumo importante, que brinda bases para el mejoramiento de las instituciones en la adherencia al Sistema de Gestión de Seguridad y Salud en el Trabajo (SGSST), la disminución de la accidentalidad laboral y fortalecer los sistemas de vigilancia epidemiológica para la prevención de enfermedades laborales.

\section{CONCLUSIONES}

Los trabajadores del área administrativa y asistencial por su naturaleza están expuestos a diferentes riesgos laborales, donde el personal administrativo tiene menor nivel de riesgo biológico laboral y presenta una mayor percepción del riesgo, evento que no sucede con los trabajadores del área asistencial, que están expuestos permanentemente al riesgo biológico y muestran una menor percepción de este.

Existe una diferencia entre los trabajadores con experiencia laboral menor o igual a 5 años y los que cuentan con mayor experiencia, en donde estos últimos tuvieron menor percepción del riesgo biológico laboral, dada la confianza adquirida a este.

La confianza es un factor primordial en la percepción del riesgo biológico laboral de la población estudiada, se observó que entre más tiempo de experiencia, menos percepción de riego del lugar de trabajo, guardando relación con la tolerancia al riesgo que se va generando al tener una exposición a estos. [34]

El perfil de riesgo aporta una información valiosa que permite descubrir claramente sobre qué variables y en qué dirección se debe enfocar la institución para lograr certeros esfuerzos en el logro de adecuados niveles de percepción.

Se sugiere realizar estudios de percepción del riesgo laboral con metodologías mixtas, para evaluar la relación entre la accidentalidad laboral y la percepción del riesgo, desde un enfoque comportamental y ser complementados así con los análisis psicométricos, pudiendo lograr explicar de forma más integral este fenómeno. 


\section{AGRADECIMIENTOS}

Se agradece especialmente al hospital por permitir la ejecución de la investigación y a todo el personal que trabaja en este. Así mismo, por su ayuda y disposición al Dr. Andrés Felipe Bustamante Orozco, encargado del Sistema de Vigilancia Epidemiológica del Riesgo Biológico y coordinador del Sistema de Gestión de Seguridad y Salud en el Trabajo de la institución.

\section{REFERENCIAS BIBLIOGRÁFICAS}

[1] Organización Internacional del Trabajo, «Riesgos emergentes y nuevos modelos de prevención en un mundo de trabajo en transformación». abr. 28, 2010, Accedido: jul. 11, 2020. [En línea]. Disponible en: https://www.ilo.org/global/topics/safety-and-health-at-work/resources-

library/publications/WCMS_124341/lang--es/index.htm.

[2] G. A. Muñoz Díaz, «Guía para trabajadores expuestos a riesgo biológico». Ministerio del Trabajo de Colombia, Accedido: jul. 11, 2020. [En línea]. Disponible en: https://www.mintrabajo.gov.co/documents/20147/59676/GUIA+RIESGO+BIOL\%C3\%93GICO+PARA+T RABAJADORES.pdf/.

[3] M. Pastrana Echeverri y M. Cifuentes Salcedo, «Conocimientos y actitudes en la aplicación de normas de bioseguridad del personal del servicio de enfermería», Rev. Colomb. Salud Ocupacional, vol. 4, n. ${ }^{\circ}$, Art. n. ${ }^{\circ}$ 1, mar. 2014, doi: 10.18041/2322-634X/rcso.1.2014.4888.

[4] L. Galíndez y Y. Rodríguez, «Riesgos Laborales de los Trabajadores de la Salud», Salud Los Trab., vol. 15, n. ${ }^{2}$ 2, pp. 67-69, 2007, Accedido: jul. 11, 2020. [En línea]. Disponible en: https://www.redalyc.org/articulo.oa?id=375839287001.

[5] D. R. Smith et al., «Organizational climate and its relationship with needlestick and sharps injuries among Japanese nurses», Am. J. Infect. Control, vol. 37, n. ${ }^{\circ}$ 7, pp. 545-550, sep. 2009, doi: 10.1016/j.ajic.2008.11.004.

[6] F. Rodríguez-Otamendi, M. R. Rodríguez-Luna, E. Soler-Huerta, A. V. Cornejo-Blanco, y E. DoloresOrdaz, «Causas de subregistro de accidentes de trabajo», Rev. Médica Inst. Mex. Seguro Soc., vol. 46, n. ${ }^{\circ}$ 5, pp. 567-570, 2008, Accedido: jul. 11, 2020. [En línea]. Disponible en: https://www.redalyc.org/articulo.oa?id=457745523018.

[7] M. De Giusti et al., «Occupational biological risk knowledge and perception: results from a large survey in Rome, Italy", Ann. Delllstituto Super. Sanità, vol. 48, pp. 138-145, jun. 2012, doi: 10.4415/ANN 12 202 06.

[8] L. M. Marsans Armengou y E. Fernández López, «Percepción del riesgo, actitudes y conducta segura de los agentes implicados en los accidentes laborales», Gest. Práctica Riesgos Laborales Integr. Desarro. Gest. Prev., n. ${ }^{\circ}$ 28, pp. 42-47, 2006, Accedido: jul. 11, 2020. [En línea]. Disponible en: https://dialnet.unirioja.es/servlet/articulo?codigo=2015544.

[9] Y. Narváez Velázquez y J. Moreno Medellín, «La percepcion de riesgos como factor causal de accidentes laborales», Gest. Práctica Riesgos Laborales Integr. Desarro. Gest. Prev., n. 99, pp. 22-26, 2012, Accedido: jul. 11, 2020. [En línea]. Disponible en: https://dialnet.unirioja.es/servlet/articulo?codigo $=4094726$.

[10] FASECOLDA, «Reporte por clase de riesgo y actividad económica». https://sistemas.fasecolda.com/rldatos/Reportes/xClaseGrupoActividad.aspx (accedido jul. 11, 2020).

[11] F. E. Montufar Andrade et al., "Accidentes ocupacionales de riesgo biológico en Antioquia, Colombia. Enero de 2010 a diciembre de 2011 », Infectio, vol. 18, n. ${ }^{\circ} 3$, pp. 79-85, sep. 2014, Accedido: jul. 11, 2020. [En línea]. Disponible en: http://www.scielo.org.co/scielo.php?script=sci_abstract\&pid=S0123$93922014000300002 \& \operatorname{lng}=e n \& n r m=i s o \& t \operatorname{lng}=e s$.

[12] J. E. Tharaldsen, E. Olsen, y T. Rundmo, «A longitudinal study of safety climate on the Norwegian continental shelf», Saf. Sci., vol. 46, n. ${ }^{\circ}$ 3, pp. 427-439, mar. 2008, doi: 10.1016/j.ssci.2007.05.006.

[13] D. Cobos Valdés, C. M. Vilariño Corella, Y. Vézquez Mojena, M. Ramos Lima, y A. Torres Valle, «Percepción del riesgo biológico en dos entidades de ciencia del sector salud en Holguín: cuba», Med. Segur. Trab., vol. 62, n. ${ }^{\circ}$ 244, pp. 212-222, sep. 2016, Accedido: jul. 11, 2020. [En línea]. Disponible en: http://scielo.isciii.es/scielo.php?script=sci_abstract\&pid=S0465-

$546 \times 2016000300005 \&$ Ing=es\&nrm=iso\&tlng=es. 
[14] Organización Mundial de la Salud OMS, «Entornos Laborales Saludables: Fundamentos y Modelo de la OMS». 2010, [En línea]. Disponible https://www.who.int/occupational_health/evelyn_hwp_spanish.pdf.

[15] C. Narocki, «Evaluación económica de la siniestralidad laboral: una aproximación a la realidad española». Instituto sindical de Trabajo, Ambiente y Salud, [En línea]. Disponible en: https://d1wqtxts1xzle7.cloudfront.net/6963653/costes.pdf?response-content-

disposition=inline\%3B+filename\%3DEVALUACION_ECONOMICA_DE_LA_SINIESTRALIDA.pdf\&Expir es=1594484633\&Signature $=$ TYH5bJd1YQrHI2PCPbp1gbRB0OfM2ft9b4D MogRP vT4rDb8t7QOUO WOj9FDKEakHEj3MQXbgajOEk-ykECi5sIR7QShvWalGQw16xemffYA-xD8PIMTPEa-jnt d58BnA80cu3DNgVjnkqkoyQpot1Eu464cxYbi28x22hDAXrDxD 7woiHzJx4s3ekLBRzQjiEAoDjvTkRwf5I G4nUu-ZzSTOYndVLRtu3r78D2bNGPtGMCYX8FRR0J58DqQmPOshJ7E8MxWPICEsnYsQggeA3SsXK6D2IScEjcMiUZleXs7J7k6t5Dga3xAvenE3TEV8 6DTWBCV8GAhek2A_\&KeyPair-Id=APKAJLOHF5GGSLRBV4ZA.

[16] J. L. Llorente Arribas, «Los costes derivados de los accidentes biológicos y su prevención», Rev. Adm. Sanit. Siglo XXI, vol. 2, n. ${ }^{\circ}$ 4, pp. 623-632, 2004, Accedido: jul. 11, 2020. [En línea]. Disponible en: https://dialnet.unirioja.es/servlet/articulo?codigo=2702091.

[17] Centro para el Control y la Prevención de Enfermedades CDC, «Datos Breves de NIOSH: Cómo prevenir las lesiones por pinchazos de aguja y objetos cortopunzantes», ene. 31, 2019. https://www.cdc.gov/spanish/niosh/docs/2012-123_sp/default.html (accedido jul. 11, 2020).

[18] Centro para el Control y la Prevención de Enfermedades CDC, «Guidelines for Safe Work Practices in Human and Animal Medical Diagnostic Laboratories». Accedido: jul. 11, 2020. [En línea]. Disponible en: https://www.cdc.gov/Mmwr/pdf/other/su6101.pdf.

[19] D. F. Gutiérrez Barona, A. Barrera Díaz, y H. A. G. Usma, «Percepción del Riesgo Laboral en Trabajadores del Área de Producción de una Empresa de Fabricación de Partes de Calzado, Santiago de Cali», Rev. Psicol. GEPU, vol. 7, n. 2, p. 7, 2016, Accedido: jul. 11, 2020. [En línea]. Disponible en: https://dialnet.unirioja.es/servlet/articulo?codigo=6919762.

[20] S. H. Bartley, Principios de percepción. Trillas, 1969.

[21] P. Slovic, B. Fischhoff, y S. Lichtenstein, «Why Study Risk Perception?», Risk Anal., vol. 2, n. ${ }^{\circ}$ 2, pp. 8393, 1982, doi: 10.1111/j.1539-6924.1982.tb01369.x.

[22] M. Portell Vidal, «NTP 578: Riesgo percibido: un procedimiento de evaluación». Ministerio del Trabajo y Asuntos Sociales de España, 1999, [En línea]. Disponible en: https://www.cso.go.cr/legislacion/notas_tecnicas_preventivas_insht/NTP\%20578\%20\%20Riesgo\%20percibido\%20un\%20procedimiento\%20de\%20 evaluacion.pdf.

[23] A. T. Carbonel Siam y A. Torres Valle, «Evaluación de percepción de riesgo ocupacional», Ing. Mecánica, vol. 13, n. ${ }^{\circ}$ 3, pp. 18-25, dic. 2010, Accedido: jul. 11, 2020. [En línea]. Disponible en: http://scielo.sld.cu/scielo.php?script=sci_abstract\&pid=S1815$59442010000300003 \& \operatorname{lng}=$ es\&nrm=iso\&tIng=es.

[24] Y. L. González, «Evaluación de la percepción del riesgo ocupacional en trabajadores de una empresa del sector de la construcción en Bogotá D.C.», NOVA, vol. 13, n. ${ }^{\circ} 23$, Art. n. ${ }^{\circ} 23$, jun. 2015, doi: $10.22490 / 24629448.1709$.

[25] C. Pozo Muñoz y E. Alonso Morillejo, «La percepción del riesgo en la prevención de accidentes laborales», Apunt. Psicol., vol. 20, n. ${ }^{3}$ 3, p. 6, 2002, Accedido: jul. 11, 2020. [En línea]. Disponible en: https://dialnet.unirioja.es/servlet/articulo?codigo $=2225364$.

[26] A. P. Rodríguez, Percepción social del riesgo: dimensiones de evaluación y predicción. Universidad Complutense de Madrid, 2001.

[27] K. Soler y A. Torres Valle, «Evaluación de percepción de riesgo aplicada a trabajadores de radioterapia». Sociedad Argentina de Radioprotección, 2015, [En línea]. Disponible en: http://www.irpabuenosaires2015.org/Archivos/trcompletos/irpa/fullpapertemplate_IRPA20153209451.pdf.

[28] Programa de las Naciones Unidas para el Desarrollo PNUD, «Objetivos de Desarrollo Sostenible», UNDP. https://www.undp.org/content/undp/es/home/sustainable-development-goals.html (accedido jul. 11, 2020).

[29] A. Torres Gómez y A. Torres Valle, «Riesgo objetivo y percepción de riesgo asociados al cáncer cervicouterino. Caso de estudio», Rev. Argent. Bioseguridad, n. 3, pp. 87-97, 2015, [En línea]. Disponible en:

https://fveter.unr.edu.ar/wpcontent/uploads/2018/09/RevistaArgentinaBioseguridadN3.pdf.

[30] B. Garea Moreda et al., Cambio Climático y Desarrollo Sostenible. Bases conceptuales para la enseñanza en Cuba. Instituto Superior de Tecnologías y Ciencias Aplicadas. Instituto Superior de Tecnologías y Ciencias Aplicadas, 2014. 
[31] A. P. López, «La Percepción social del riesgo: Algo más que discrepancia expertos/público», Nucleus, vol. 0, n. ${ }^{\circ}$ 26, Art. n. ${ }^{\circ}$ 26, 1999, Accedido: jul. 11, 2020. [En línea]. Disponible en: http://nucleus.cubaenergia.cu/index.php/nucleus/article/view/329.

[32] A. T. Carbonell Siam, A. Torres Valle, Y. Nuñez Valdivie, y Á. M. Aranzola Acea, «Análisis de percepción de riesgos laborales de tipo biológico con la utilización de un sistema informático especializado», Rev. Cuba. Farm., vol. 47, n. ${ }^{\circ}$ 3, pp. 324-338, sep. 2013, Accedido: jul. 11, 2020. [En línea]. Disponible en: http://scielo.sld.cu/scielo.php?script=sci_abstract\&pid=S0034$75152013000300005 \& \operatorname{lng}=\mathrm{es} \& n r m=i s o \&$ tIng=es.

[33] J. M. Rodríguez Rueda, V. González Ferrer, T. Montero González, y A. N. Consuegra Carvajal, "Regresión logística binaria para crear un modelo predictivo de daño hepático en el paciente séptico», Acta Médica Cent., vol. 12, n. 1, pp. 10-18, 2018, Accedido: jul. 11, 2020. [En línea]. Disponible en: https://www.medigraphic.com/cgi-bin/new/resumen.cgi?IDARTICULO=77321.

[34] E. Arellano, L. Camarena, C. von Glascoe, y W. Daesslé, «Percepción del riesgo en salud por exposición a mezclas de contaminantes: el caso de los valles agrícolas de Mexicali y San Quintín, Baja California, México», Rev. Fac. Nac. Salud Pública, vol. 27, n. 3, pp. 291-301, sep. 2009, Accedido: jul. 11, 2020. [En línea]. Disponible en: http://www.scielo.org.co/scielo.php?script=sci_abstract\&pid=S0120$386 \times 2009000300006 \& \operatorname{lng}=e \& n r m=i s o \&$ tlng $=e s$.

[35] J. Mullen, «Investigating factors that influence individual safety behavior at work», J. Safety Res., vol. 35, n. ${ }^{\circ}$ 3, pp. 275-285, 2004, doi: 10.1016/j.jsr.2004.03.011. 